\title{
Estrategias y retos para el seguimiento educativo en primarias ante la contingencia covid-19 en Sonora, México
}

\author{
Strategies and challenges for educational monitoring in elementary schools \\ in the face of the COVID-19 contingency in Sonora, Mexico
}

\begin{abstract}
Estratégias e desafios para o monitoramento educacional em escolas de ensino fundamental em face da contingência COVID-19 em Sonora, México
\end{abstract}

\author{
Reyna de los Ángeles Campa Álvarez \\ Universidad de Sonora, México \\ reyna.campa@unison.mx \\ https://orcid.org/0000-0003-3780-9623
}

\section{Resumen}

La emergencia sanitaria actual y el confinamiento prolongado causados por la covid-19 han trastocado las diversas áreas de la vida, entre ellas, la educativa, lo que ha provocado un reajuste en las estrategias de enseñanza-aprendizaje para la continuidad del ciclo escolar. Por ello, el objetivo general de la presente investigación fue identificar las estrategias educativas empleadas por los docentes de nivel de primaria en el estado de Sonora (México) para el seguimiento escolar ante la pandemia covid-19, así como enunciar los principales retos experimentados en tal proceso. El enfoque de investigación fue cualitativo de tipo exploratorio; los participantes fueron 42 docentes de primarias públicas a quienes se les aplicó un cuestionario online en formato de entrevista con preguntas abiertas. Los principales resultados muestran que las plataformas digitales, las videoconferencias y las redes sociales son las más usadas como estrategias de la educación a distancia. En el caso de los estudiantes que no tienen acceso a internet o a equipo de cómputo, los docentes usan llamadas a los padres de familia, seguimiento del programa televiso Aprende en casa y entrega de material impreso. Asimismo, se emplean estrategias tradicionales como el portafolio de evidencias, ejercicios del libro de texto y exámenes. Los principales retos detectados fueron la búsqueda 

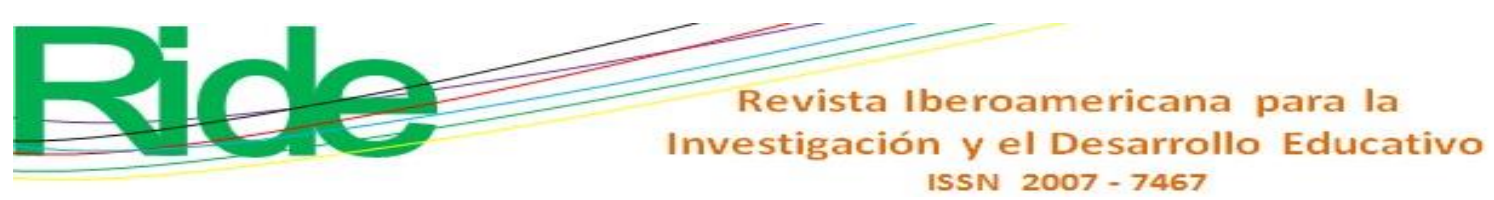

\section{Resumo}

A atual emergência de saúde e o confinamento prolongado derivado do COVID-19 perturbou as várias áreas da vida, incluindo a educação; causando um reajuste nas estratégias de ensinoaprendizagem para a continuidade do ano letivo antes da suspensão das aulas presenciais. $\mathrm{O}$ objetivo geral da pesquisa foi identificar as estratégias educacionais utilizadas por professores do ensino fundamental para o acompanhamento escolar em face da pandemia covid-19, bem como apresentar os principais desafios enfrentados em tal processo no estado de Sonora, México. A abordagem da pesquisa foi qualitativa, exploratória, um questionário online com questões abertas foi aplicado a 42 professores do ensino fundamental da rede pública. Os principais resultados mostram que dentro das estratégias utilizadas se deu por meio da implantação da educação a distância por meio de plataformas digitais, videoconferências e uso de redes sociais. No caso dos alunos que não tinham acesso à internet ou equipamento de informática, foram realizadas ligações para os pais, acompanhamento do programa de televisão Aprende en Casa e entrega de material impresso; bem como continuar com estratégias tradicionais, como o portfólio de evidências, exercícios de livros e testes. Os principais desafios foram a busca de canais de comunicação para os alunos em situação de vulnerabilidade; bem como as dificuldades dos pais no acompanhamento das tarefas e exercícios dos filhos e filhas. Conclui sobre a importância da formação contínua para o uso das TIC, o envolvimento dos pais no processo educativo e por parte do Estado para viabilizar recursos tecnológicos a alunos e professores.

Palavras-chave: estratégias educacionais, educação a distância, tecnologia educacional, adaptação escolar, educação básica.

Fecha Recepción: Noviembre 2020

Fecha Aceptación: Mayo 2021

\section{Introducción}

Los cambios derivados en materia de educación por la covid-19 han transformado los métodos de enseñanza-aprendizaje, pues se han tenido que implementar estrategias pedagógicas mediadas por la tecnología para continuar con las actividades académicas de manera virtual. Una investigación realizada por Baptista, Almazán, Loeza, López y Cárdenas (2020) señala que los docentes de nivel básico del sector público han utilizado herramientas digitales combinadas con recursos tradicionales (libro de texto) para el seguimiento escolar 


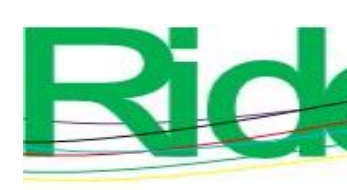

Revista Iberoamericana para la
Investigación y el Desarrollo Educativo
ISSN $2007-7467$

durante la pandemia, aunque al mismo tiempo demuestra la falta de acceso a la tecnología en los hogares.

Por su parte, Gazca (2020) realizó una investigación de tipo no experimental, exploratoria, descriptiva y cuantitativa con el objetivo de diagnosticar las consecuencias que ha tenido la covid-19 sobre los procesos de enseñanza en la educación superior. Los resultados evidencian que los docentes cuentan con una plataforma institucional e infraestructura tecnológica para impartir clases, y demuestran que existe una asociación entre las variables edad y capacitación tecnológica para dar clases en línea, que y la edad de los docentes se relaciona con las afectaciones en la salud. Además, los hallazgos reflejan la incertidumbre generada por la prolongación del confinamiento.

Por otra parte, el trabajo de Cifuentes (2020) enseña que docentes y discentes han tenido que adaptarse rápidamente a la educación a distancia, situación que demanda una mayor formación y una visión distinta para la incorporación de estrategias que capten la atención del estudiante en un sistema virtual. Cabero y Valencia (2021) refieren que el confinamiento ha impactado de una manera acelerada a los sistemas educativos de todos los niveles al reemplazar repentinamente las clases presenciales por una modalidad en línea. Este autor señala la brecha digital existente no solamente en acceso a la tecnología, sino en el conocimiento y uso de esta para fines educativos.

Como se pude inferir de los párrafos anteriores, las tecnologías de la información y comunicación (TIC) se han convertido en herramientas esenciales para continuar con las actividades académicas; no obstante, se presentan una serie de barreras que limitan el acompañamiento a los estudiantes, las cuales se vinculan con una marcada desigualdad social, falta de recursos tecnológicos, y escasa capacitación de docente y discentes en uso de las TIC (Sánchez et al., 2020). Esta situación afecta principalmente a las poblaciones vulnerables, con baja escolarización y trabajo informal, las cuales tienen menos posibilidades de acceder a una educación a distancia (Ducoing, 2020).

En México, de acuerdo con cifras del Consejo Nacional de Evaluación de la Política de Desarrollo Social [Coneval], en el año 2018 más de 52 millones de personas se encontraban en una situación de pobreza, 36 millones fueron consideradas como población vulnerable debido a carencias sociales, y 21 millones presentaron como indicador el rezago educativo. De hecho, según el Instituto Nacional de Estadística y Geografía [Inegi] (2020), se estima que $76 \%$ de la población en México utiliza internet en zonas urbanas, mientras que en las rurales solo $47 \%$ lo hace. Asimismo, mientras que $92 \%$ de los hogares cuentan con 


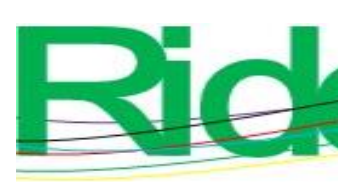

Revista Iberoamericana para la
Investigación y el Desarrollo Educativo
ISSN $2007-7467$

televisión, solo $56 \%$ tiene acceso a internet, $88 \%$ cuenta con al menos un celular inteligente y únicamente $44 \%$ dispone de una computadora. En el caso concreto de Sonora, se estima que $60 \%$ de los hogares tienen acceso a internet y cuentan con computadora.

Esta realidad latente en México — y en varios países del mundo- constituye una gran brecha digital para alcanzar las metas educativas, de ahí que sea necesario reflexionar sobre los esquemas de educación a distancia y las estrategias educativas utilizadas para facilitar la inclusión escolar de los sectores más vulnerables.

En virtud de lo anterior, el objetivo del presente trabajo fue identificar las principales estrategias empleadas, así como los retos asumidos por los docentes de nivel primaria del estado de Sonora (México) para continuar con sus actividades escolares en medio del aislamiento social generado por la covid-19. Para ello, se plantearon las siguientes preguntas: ¿qué estrategias educativas implementaron los docentes para el seguimiento escolar?, y ¿cuáles fueron los principales retos educativos encarados ante la covid-19?

Para responder dichos cuestionamientos se formularon las siguientes hipótesis:

- H1: La implementación de una educación a distancia permite el seguimiento escolar ante el confinamiento.

- H2: Las estrategias didácticas tradicionales permiten incluir a los sectores más vulnerables.

- H3: Los principales retos educativos son las brechas digitales de docentes y dicentes.

\section{Marco conceptual}

La emergencia sanitaria generada por la covid-19 ha provocado un reajuste de las actividades escolares, las cuales han tenido que cambiar repentinamente hacia una educación a distancia (Moreno, 2020), entendida como el proceso de enseñanza-aprendizaje que emplea medios para la interactividad síncrona (teléfono, chat, videoconferencia, etc.) y asincrónica (plataformas, foros, correo electrónico, etc.) (Heedy y Uribe, 2008).

Una de las características más resaltantes de la educación a distancia es el protagonismo que debe asumir el estudiante en la construcción de su conocimiento, lo cual es fomentado por la tutoría de los docentes a través de una interacción virtual (Chaves, 2017). En esta modalidad se requiere de un proceso de planeación, selección de recursos, estrategias educativas y formas de comunicación mediadas por las TIC (Vela, Ahumada y Guerrero, 2015), las cuales se pueden definir como el conjunto de tecnologías que permiten la 


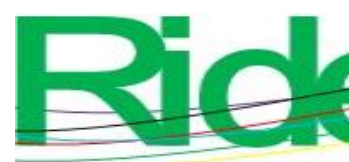

Revista Iberoamericana para la
Investigación y el Desarrollo Educativo
ISSN $2007-7467$

adquisición, producción, almacenamiento, tratamiento, comunicación, registro y presentación de informaciones en forma de voz, imágenes y datos (Cacheiro, 2014).

Al respecto, Mendoza (2018) agrega que las TIC ofrecen herramientas claves para el desarrollo de materiales digitales en la acción educativa, los cuales deben ser diseñados tomando en cuenta los objetivos trazados, así como los temas y la población a la cual van dirigidos (Moreira, 2019). Por ende, Cervantes y Gutiérrez (2020) destacan la importancia de fomentar las habilidades requeridas para el uso de esas tecnologías (p. ej., la autorregulación del aprendizaje), particularmente cuando se trabaja con estudiantes de educación básica, pues de ese modo se pueden reducir los índices de exclusión y rezago escolar.

Previendo estos problemas, la Secretaría de Educación Pública (SEP) de México diseñó dos estrategias claves para brindar continuidad académica en medio del aislamiento social generado por la covid-19:

Aprende en casa: Consiste en la transmisión de programas televisivos para explicar temas relacionados con cada grado escolar. Aunado a esto, se brinda la posibilidad de acceder al portal de dicho programa para consultar actividades, contenido y videos de YouTube (SEP, 2020a).

Estrategia de educación a distancia: transformación e innovación para México. Esta opera a partir de cuatro ejes: 1) establecimiento de la plataforma a distancia para la SEP, 2) contenidos (materias, con métodos, lúdicos, auditivos y visuales), 3) capacitación y acompañamiento a docentes, padres de familia, estudiantes y funcionarios, y 4) métricas de uso como insumo para su posible monitoreo y evaluación (SEP, 2020b).

Ahora bien, en cuanto a las estrategias didácticas, Tobón (2010) las define como un conjunto de acciones que se diseñan e implementan de forma ordenada para lograr los aprendizajes esperados, mientras que Ferreiro (2012) las concibe como herramientas de mediación entre el estudiante y los contenidos facilitados por el docente.

Sin embargo, Díaz-Barriga y Hernández (2010) explican que no se puede agrupar en una misma definición a las estrategias de enseñanza y aprendizaje, pues las referidas a la enseñanza aluden a procedimientos usados por el docente para promover y facilitar el aprendizaje significativo de los estudiantes, mientras que las de aprendizaje se vinculan con las empleadas de manera personal por los estudiantes para reconocer, aprender y aplicar la 


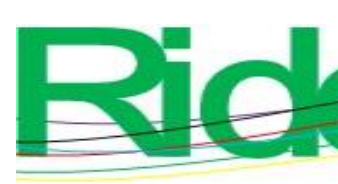

Revista Iberoamericana para la
Investigación y el Desarrollo Educativo
ISSN $2007-7467$

información recibida, de ahí que los docentes deban manejar una variedad de actividades para atender a esas particularidades.

\section{Materiales y método}

El enfoque de investigación del presente trabajo fue cualitativo, pues se ha hecho énfasis en las perspectivas de las personas y en cómo son interpretadas en un contexto particular (Vasilachis, 2006). Al respecto, Maxwell (2004) afirma que la investigación cualitativa suele utilizarse para comprender los significados que los actores dan a sus acciones, vidas, experiencias, procesos y situaciones en las que participan. Por su parte, Denzin y Lincoln (2012) señalan que en este tipo de indagaciones se emplea el multimétodo focalizado para el entendimiento e interpretación de fenómenos sociales en términos del significado que otorgan las personas.

Asimismo, se puede acotar que esta es una investigación exploratoria porque solo se ofrece un primer acercamiento a las estrategias empleadas y a los retos educativos que deben encarar docentes del nivel de primaria del sector público en México producto del aislamiento provocado por la covid-19. Este tipo de estudios — según Hernández, Fernández y Baptista (2010) - procuran examinar un tema reciente para detectar variables promisorias que serán detalladas en trabajos futuros. Desde el enfoque cualitativo se persigue estudiar un número reducido de casos extraídos de un segmento poblacional con el objeto de comprender un hecho social (Izcara, 2014).

Por otra parte, en cuanto a la muestra, esta fue no probabilística intencional por conveniencia, conformada por 42 docentes (78.6\% mujeres y $21.4 \%$ hombres) de educación primaria del sector público del estado de Sonora (México), con una antigüedad laboral que oscilaba entre 1 y 30 años.

Los criterios de selección establecidos fueron los siguientes: a) docentes de primarias activos en escuelas públicas y b) disposición para participar en la investigación y compartir su experiencia. Asimismo, los códigos de identificación fueron estos: número de profesor participante $(\mathrm{P \#})$; sexo del participante $(\mathrm{H}=$ hombre; $\mathrm{M}=$ mujer $)$ y grado educativo que imparte (G\#). Por tanto, el código P1_M_G1 significaría participante uno, mujer, grado primero.

Para la recolección de datos se empleó un cuestionario — en formato de entrevista con preguntas abiertas - diseñado exprofeso para fines de la investigación. El primer apartado 

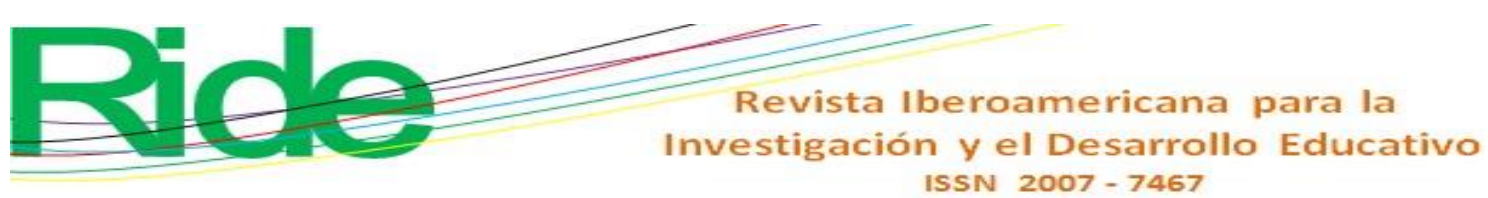

se enfocó en los siguientes datos: 1) sexo, 2) antigüedad docente, 3) nivel de estudios de los docentes, 4) grado educativo que imparte y 5) tipos de estudiantes atendidos durante el ciclo escolar (acceso a las TIC, dispositivo móvil inteligente, sin acceso a internet o computadora). En el segundo apartado se presentaron cuatro preguntas abiertas, las cuales se estructuraron según las siguientes categorías (tabla 1):

Tabla 1. Categorías y preguntas de investigación

\begin{tabular}{|l|l|}
\hline Categorías & Preguntas \\
\hline $\begin{array}{l}\text { 1) Estrategias educativas } \\
\text { adaptadas para el trabajo } \\
\text { no presencial. }\end{array}$ & $\bullet \begin{array}{l}\text { ¿Qué estrategias implementó para la } \\
\text { continuidad académica? (Narre su experiencia } \\
\text { de acuerdo con el tipo de estudiante). }\end{array}$ \\
\hline 2) $\begin{array}{l}\text { Modalidades de } \\
\text { inclusión y seguimiento. }\end{array}$ & $\begin{array}{l}\text { ¿Cuáles fueron las estrategias para el } \\
\text { seguimiento de los estudiantes con y sin } \\
\text { necesidades educativas especiales? (Narre su } \\
\text { experiencia). }\end{array}$ \\
¿Qué modalidades y formas de trabajo se \\
implementaron para la inclusión de los \\
estudiantes?
\end{tabular}

Fuente: Elaboración propia

La recolección de datos se concretó entre mayo y octubre de 2020 mediante un formulario de Google Forms. La duración aproximada para las respuestas fue de 20 minutos. Primero se contactó a los docentes de educación primaria del sector público mediante una invitación y luego se solicitó su autorización para colaborar en la investigación (se garantizó el anonimato y la confidencialidad). La información recabada se descargó del vaciado de datos automático de Google Forms y se exportó al procesador de texto de Word. Posteriormente, se construyó la unidad hermenéutica en el programa Atlas ti (versión 7), donde se procesó la información, se asignaron códigos a los testimonios recopilados, se realizaron categorías de análisis y memos con anotaciones de la saturación del discurso de los participantes, y finalmente se construyeron familias y redes de relación. 


\section{Resultados}

Los resultados indican que, ante la contingencia sanitaria generada por la covid-19, los docentes han utilizado diversas estrategias educativas, las cuales dependían del acceso que los estudiantes tuvieran a las TIC. En tal sentido, se identificó que 1) $60 \%$ de los alumnos contaba con computadora e internet, 2) $80 \%$ con internet y dispositivo móvil inteligente, y 3) $15 \%$ no contaba con internet o computadora. A partir de estas tres categorías, en la figura 1 se enseñan las estrategias implementadas con cada grupo:

Figura 1. Estrategias educativas implementadas por tipo de estudiantes

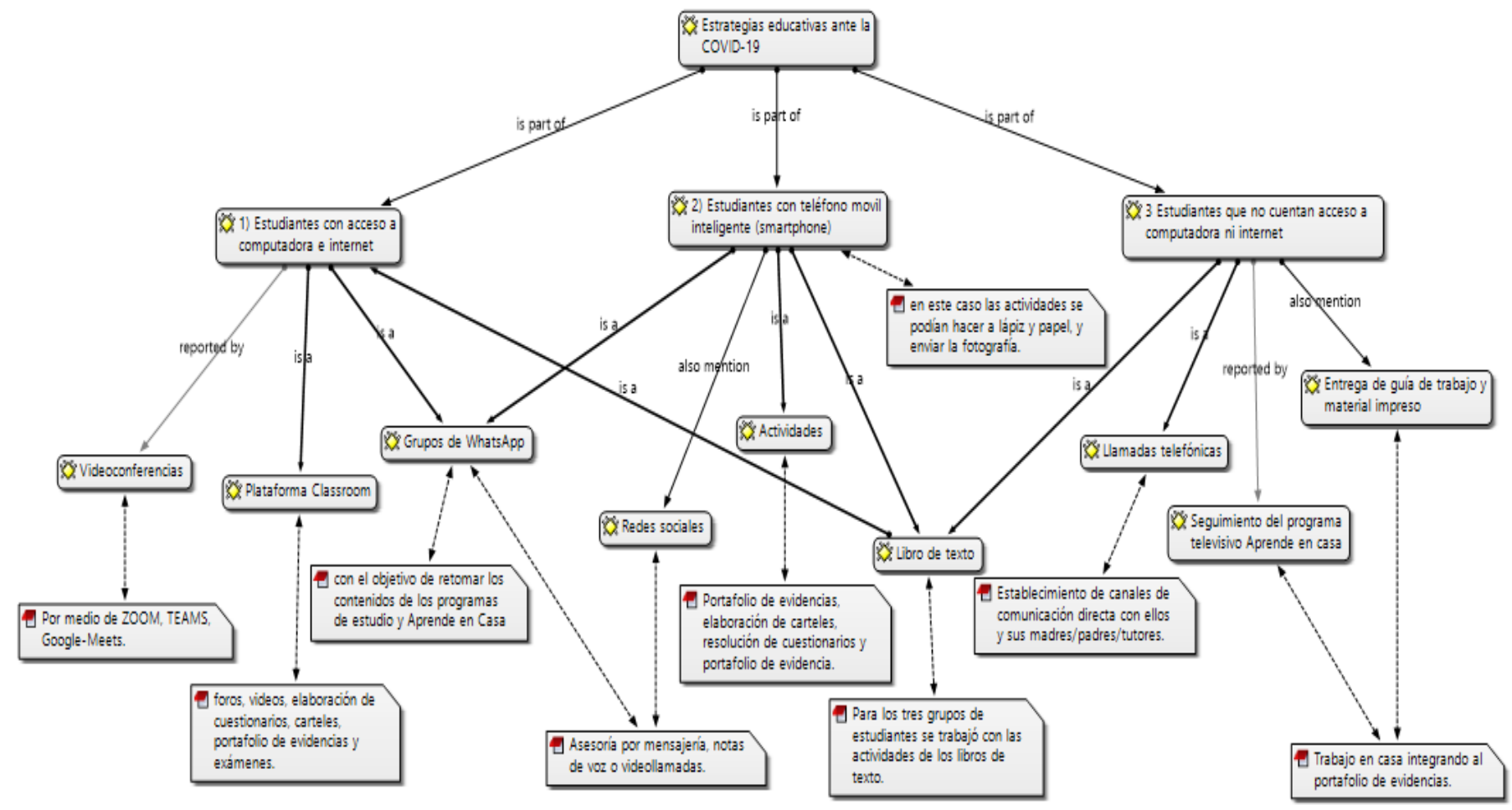

Fuente: Elaboración propia

En la red anterior se aprecia que para el primer grupo de estudiantes (con acceso a computadora e internet) se utilizó principalmente como medio de comunicación sincrónica (remota en tiempo real) las videoconferencias por medio de plataformas como Zoom, Teams y Google-Meets. Asimismo, se crearon grupos de WhatsApp con el objetivo de brindar instrucciones, asesorías y retomar las actividades del programa televisivo Aprende en casa.

El seguimiento asíncrono, en cambio, se realizó a través de Classroom, con actividades como foros, videos de YouTube y elaboración de cuestionarios. Al respecto, los profesores comentaron: 


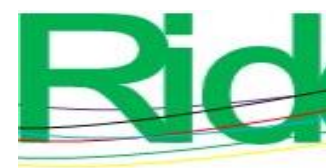

Revista Iberoamericana para la
Investigación y el Desarrollo Educativo
ISSN $2007-7467$

Hasta ahora se han impartido clases vía Zoom, en donde se han reproducido videos, exposición, exámenes en línea y exposición por parte de los alumnos (P7_H_G5).

La comunicación y trabajo que se llevó con la mayoría de los jóvenes fue por medio de Classroom, la cual nos facilitó el contacto con los alumnos y por este medio trabajar; y también trabajé con papás mandando actividades por WhatsApp para los jóvenes que no contaban con los medios (P17_H_G6).

En cuanto al segundo grupo de estudiantes (con teléfono móvil inteligente), como medio de comunicación sincrónica se usaron los grupos de WhatsApp y la mensajería instantánea de redes sociales como Facebook. Sobre estas estrategias los docentes expresaron:

En los grupos de WhatsApp se daba la instrucción a todos los alumnos, se les explicaba por texto y mandaba audios para resolver dudas de los papás, en algunos casos me escribía directamente la mamá y hacia videollamada (P16_M_G3).

Tuve que realizar grupos de Facebook porque los papás sugirieron que era más sencillo ver los archivos y seguir las actividades, y para contactarme directamente con ellos (P9_M_G4).

A este grupo de estudiantes también se solicitó que siguieran el programa televisivo Aprende en casa y efectuaran las actividades del libro de texto, las cuales podían ser realizadas a lápiz y enviadas en fotografía por WhatsApp:

El envío de las tareas era por WhatsApp mediante las fotos de los trabajos realizados del libro (P8_M_G5).

La comunicación se dio por medio de los celulares de padres de familia, se les enviaban las actividades cada domingo para realizar en la semana y los viernes ellos enviaban fotos de evidencias del trabajo realizado (P20_M_G3).

Por otra parte, para el tercer grupo de estudiantes (los que no contaban con acceso a computadora e internet), los docentes entregaron material impreso y cuadernillos de trabajo:

Se trabajó con los libros de texto, mandando la información a un lugar específico, como un establecimiento (P29_H_G4).

Les pedí que realizaran ellos actividades de repaso y que utilizaran sus libros de texto o libros de trazos (P7_M_G1). 


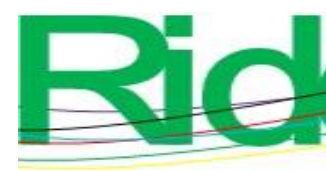

Revista Iberoamericana para la
Investigación y el Desarrollo Educativo
ISSN $2007-7467$

Los ubiqué en sus domicilios y dejé copias en la papelería más cercana con ejercicios de matemáticas y español (P21_ M_G2).

Igualmente, se usaron las llamadas telefónicas como medio de contacto:

En estos casos en un inicio fue complicado, primero en contactar a los papás, conseguir los celulares, y hacerles llamadas de monitoreo cada semana para mantener la comunicación (P18_M_G5).

Por último, los docentes refirieron que emplearon la estrategia educativa nacional del programa televisivo Aprende en casa:

Les expliqué a los papás de la importancia de que vieran el programa por TV Aprende en casa para que el niño fuera al corriente y daba monitoreo por llamadas (P27_H_G3).

La sugerencia principal fue que vieran el programa Aprende en casa y que hicieran un horario como si fueran a clases regulares (P15_M_G6).

En la categoría de modalidades de inclusión y seguimiento académico para los estudiantes con necesidades educativas especiales (NEE), los resultados refieren la impartición de una educación a distancia:

Se han atendido por medio de redes sociales tales como WhatsApp, Zoom y correo electrónico (P6_H_G3).

Se implementan sesión de clase por Zoom con instrucciones para ejecutar las actividades en casa (P10_H_G2).

En estos casos, además, se realizó una planeación personalizada con los estudiantes mediante el seguimiento por celular y explicando las actividades del cuadernillo de trabajo:

El seguimiento se dirigió a todos los estudiantes, pero las herramientas que se implementaron con los niños con necesidades especiales fueron atención personalizada, dándole un día para atender a dicho alumno (P31_H_G3)

Una alumna que estaba en proceso de alfabetización se le envió un cuadernillo adecuado a su nivel de alfabetización, se mantuvo una constante comunicación con los padres para dar sugerencias de actividades (P12_M_G1).

Un elemento destacado en todo este proceso fue la participación de los padres de familia y el seguimiento del personal de apoyo: 
Al principio se les dificultó un poco seguir con la programación de las clases en su hogar, pero después fuimos trabajando con una planeación semanal que les ayudó a seguir repasando y aprendiendo nuevos temas (P14_M_G5).

Les envié actividades adecuadas a sus necesidades, así como videos hechos por mí, de ejercicios para trabajar motricidad fina, gruesa y lenguaje (P23_M_G1).

Trabajo en conjunto con los profesionales maestra de USAER, psicóloga, trabajadora social y maestra de apoyo (P4_M_G3).

En la figura 2 se agrupan los resultados sobre las modalidades de inclusión y seguimiento académico:

Figura 2. Modalidades de inclusión y seguimiento académico

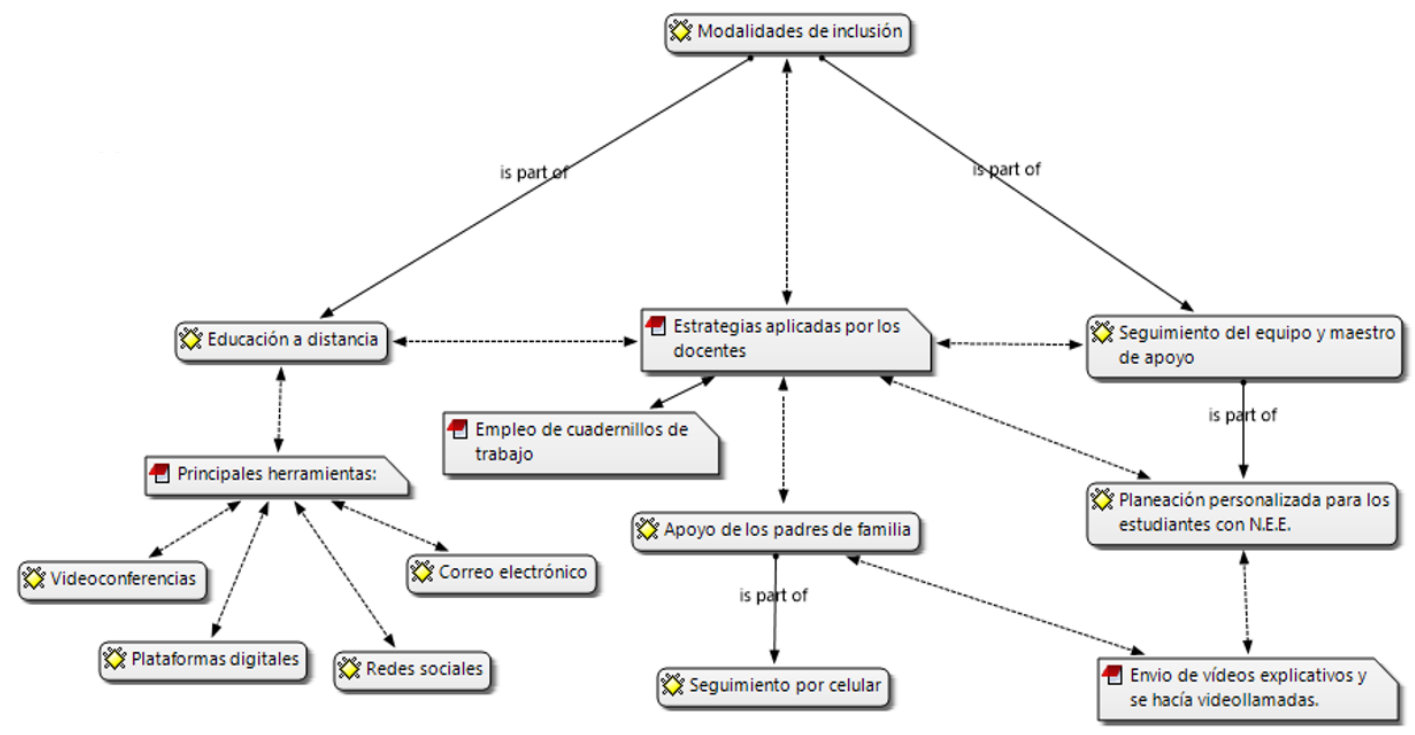

Fuente: Elaboración propia

En relación con los principales retos educativos experimentados ante la contingencia covid-19, en la figura 3 se agrupan los resultados. 
Figura 3. Principales retos educativos experimentados ante la contingencia covid-19 (percepción docente de primaria)

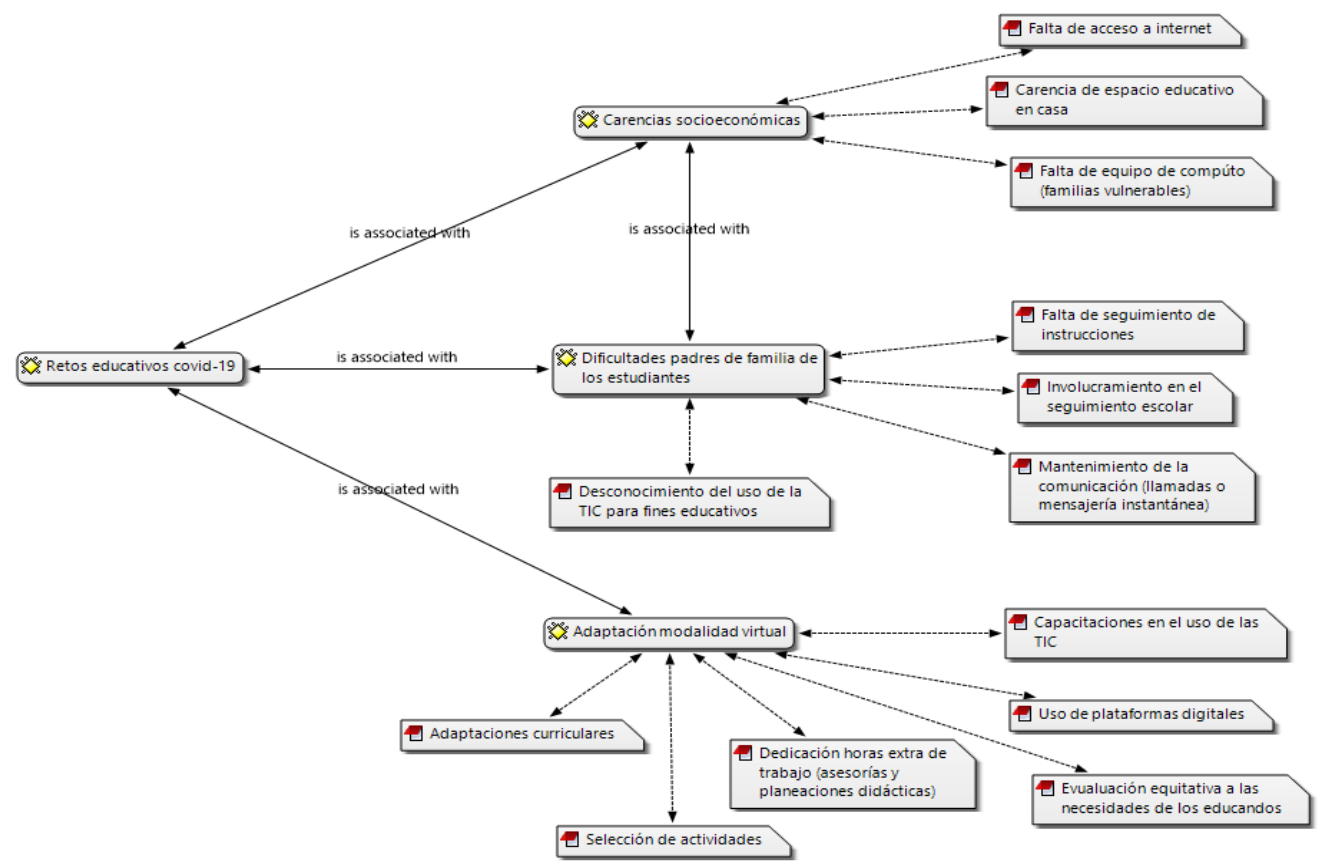

Fuente: Elaboración propia

En los siguientes fragmentos se reflejan los principales retos según la percepción de los docentes:

Es una situación muy difícil y nunca vista, educación a distancia. Pocos han sido los padres que han cumplido con su labor, quiero pensar que existen varios factores que limitan el trabajo del padre. Tal es la situación económica por la que atraviesan, o la falta de conocimiento de cómo enseñar, aunque muchos desempeñaron su labor al pie de la letra. Y estuvieron siempre dispuestos a ayudar (P15_H_G4).

El trabajar a distancia sin duda es más complicado, pero logramos adaptarnos a esta modalidad muy rápido, no todos aprenden a la par, por lo cual hay que regresarnos al contenido y hacer adecuaciones (P7_M_G6). 


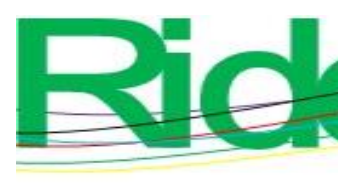

Revista Iberoamericana para la Investigación y el Desarrollo Educativo ISSN 2007-7467

\section{Discusión}

En cuanto a la primera pregunta de investigación formulada en este trabajo (¿Qué estrategias educativas implementaron los docentes para el seguimiento escolar?), los resultados señalan una transformación del trabajo metodológico, así como del uso de las TIC en el proceso de enseñanza-aprendizaje, lo cual constituyó una oportunidad para innovar en la práctica docente. En tal sentido, y siguiendo la estrategia nacional de educación a distancia propuesta por la SEP, los docentes se apoyaron en plataformas como Google Classroom y en videoconferencias que facilitaron por Google Meets, entre otras.

En este sentido, la figura del docente se materializa en un profesional en permanente formación que innova en su práctica empleando instrumentos y recursos que se ajusten a las necesidades de los estudiantes (Muñoz y Lluch, 2020; Zavala, González y Vázquez, 2020). Asimismo, Parra, Gómez y Pintor (2015) mencionan que el uso de las TIC en el proceso educativo implica una transformación del trabajo metodológico y la formación de los docentes para enfrentar los retos que demanda la sociedad actual.

En cuanto a la hipótesis dos (Las estrategias didácticas tradicionales permiten incluir a los sectores más vulnerables), se puede señalar que un número reducido de estudiantes no tiene acceso a las TIC, lo cual genera una desventaja y brecha digital. Aun así, los resultados demuestran la vocación docente y el compromiso del personal involucrado, los cuales efectuaron adecuaciones curriculares y ofrecieron otras posibilidades para que este grupo de alumnos continuara con su proceso de aprendizaje. De hecho, se destaca la dedicación de los docentes, quienes brindaron asesoría incluso fuera de los horarios establecidos, grabaron videos explicativos y entregaron material impreso para que avanzaran desde sus casas. Otro punto relevante fue el apoyo de los padres de familia para mediar los aprendizajes y continuar con las actividades del programa televisivo Aprende en casa, aunque cabe apuntar que se requiere mayor participación de estos.

Por eso, es indispensable que los docentes establezcan un trabajo coordinado con padres de familia, maestros de apoyo y directivos para facilitar la realización de las tareas y actividades académicas (Cáceres, 2020; García, 2012). Además, como explican Burgos, Vázquez, López y Adaos (2021), se deben pensar en la posibilidad de reformar el currículo para implementar formas de evaluación ajustadas a las necesidades de los estudiantes, promover mayor participación de los padres de familia en el proceso educativo e impulsar un aprendizaje cooperativo. Asimismo, como sugieren Carmona y Morales (2021), se debe 


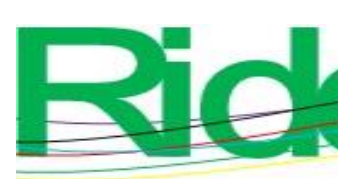

Revista Iberoamericana para la
Investigación y el Desarrollo Educativo
ISSN $2007-7467$

trabajar en la combinación de la educación a distancia y la tradicional mediante el uso de plataformas virtuales y programas televisivos abiertos, lo cual ofrecerá más cobertura educativa.

Por otra parte, en relación con la hipótesis tres (Los retos educativos son las brechas digitales de docentes y dicentes), los hallazgos obtenidos demuestran que los principales desafíos son los siguientes: adecuación de estrategias a la modalidad virtual (uso de plataformas y selección de actividades), capacitación docente en el uso de las TIC, dificultades con padres de familia y estudiantes en el uso de las TIC, falta de equipo de cómputo e internet (familias vulnerables), comunicación con padres de familia y estudiantes.

Estos resultados concuerdan con una investigación realizada por Sánchez et al. (2020), donde se evidencia que los docentes presentan problemas logísticos, tecnológicos, así como brechas digitales, por lo que sugieren adoptar actitudes positivas y desarrollar estrategias creativas e innovadoras para enfrentar la situación actual. Al respecto, Umaña (2020) enuncia las principales situaciones que han impactado en el área educativa: la falta de recursos tecnológicos y didácticos para implementar un modelo de educación a distancia, la abrupta utilización de las TIC y la necesidad de fortalecer el desarrollo de competencias digitales.

En lo concerniente a las limitaciones de la presente investigación, en un primer momento se esperaba concretar entrevistas a profundidad con los docentes por medio de videoconferencias. Sin embargo, no se pudo compaginar los horarios, por lo cual se optó por realizar preguntas abiertas y enviarlas para que las contestaran en línea desde sus casas. El segundo aspecto fue que se esperaba un mayor número de participantes; no obstante, al tener restricciones espaciales y temporales, se presentaron dificultades para ampliar este número.

Asimismo, es conveniente referir que se optó por un tipo de muestreo no probabilístico intencional por conveniencia debido a la característica de la población participante (docentes de primarias públicas), la cual tuvo un exceso de actividades laborales y personales en medio de la pandemia.

Finalmente, el Estado debe considerar el desarrollo de estrategias para que docentes, discentes y padres de familia se capaciten y tengan acceso al uso de herramientas digitales, pues solo de esa manera se podrá impulsar una educación a distancia efectiva y de calidad. 


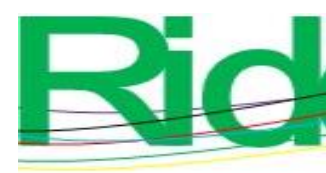

Revista Iberoamericana para la Investigación y el Desarrollo Educativo ISSN 2007-7467

\section{Conclusiones}

Los resultados derivados de la investigación permiten señalar que los docentes de educación primaria de Sonora (México) utilizan videoconferencias y mensajería instantánea (medios de comunicación síncrona), así como plataformas digitales (recursos asincrónicos) para continuar con el desarrollo en línea de las actividades académicas en medio de la emergencia sanitaria provocada por la covd-19. Asimismo, también se apoyan en estrategias nacionales como el programa de televisión Aprende en casa para procurar atender a los sectores más vulnerables que no tienen acceso a las TIC, lo cual demuestra que aún persisten las desigualdades sociales.

Por otra parte, vale destacar el esfuerzo, el ingenio y el compromiso de los docentes con su misión formativa, pues en medio de las dificultades procuran crear estrategias de diversa índole para que todos los estudiantes continúen con sus actividades de aprendizaje. Esto se evidenció en la forma como combinaron métodos tradicionales (p. ej., el uso del libro de texto) y virtuales (p. ej., videos) para que los alumnos de menores recursos no fueran excluidos ni quedaran rezagados. Además, en el caso de los estudiantes con necesidades educativas especiales (NEE), trabajaron en conjunto con el equipo de apoyo y continuamente se mantuvo una comunicación directa con los padres de familia para el cumplimiento de las actividades. Igualmente, se hicieron adecuaciones curriculares, planeaciones semanales y se siguió utilizando el cuadernillo de trabajo.

En cuanto a las debilidades encontradas, se pueden referir los escasos conocimientos tecnológicos de los padres para brindar asesorías a los hijos, lo que es una evidencia de las brechas digitales existentes. Por eso, se deben impulsar estrategias de formación en el uso de las TIC para fines educativos, así como ofrecer materiales y recursos tecnológicos para los más necesitados.

Sobre los retos educativos derivados de la contingencia sanitaria, se pueden agrupar tres: 1) económicos, relacionados con las carencias sociales y de acceso a las TIC por parte de los estudiantes; 2) familiares, vinculados con las dificultades de los padres de familia para el acompañamiento académico y regulación de los aprendizajes, y 3) adaptación al trabajo virtual, lo cual tiene que ver con la modificación de las estrategias didácticas para ser empleadas en espacios digitales. 

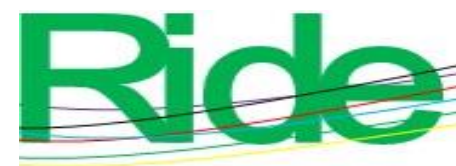

Revista Iberoamericana para la Investigación y el Desarrollo Educativo

ISSN $2007-7467$

Finalmente, cabe señalar que hoy más que nunca es necesario adoptar una actitud innovadora para el próximo ciclo escolar. En estos momentos se viven situaciones inesperadas que han provocado cambios emocionales, económicos, educativos, etc., que deben ser encarados por docentes capacitados y empáticos que brinden acompañamiento en las emergentes necesidades de la población estudiantil.

\section{Futuras líneas de investigación}

Para futuros trabajos se propone estudiar a través de un enfoque cuantitativo las variables 1) formación docente inicial y continua en educación a distancia y 2) competencias digitales educativas de docentes, discentes y padres de familia. Asimismo, se deben desarrollar investigaciones dedicadas exclusivamente a los estudiantes con necesidades educativas especiales y al equipo de apoyo durante el confinamiento. Por último, se sugiere realizar entrevistas con mayor profundidad a los docentes e incluir en la investigación a los padres de familia y a los estudiantes para recopilar datos de estos sectores involucrados en el proceso educativo.

\section{Agradecimientos}

Esta investigación fue realizada gracias al apoyo y financiamiento del Programa para el Desarrollo Profesional Docente (Prodep) (tipo superior). Secretaría de Educación Pública (SEP). Folio del proyecto: Unison-PTC-297. 


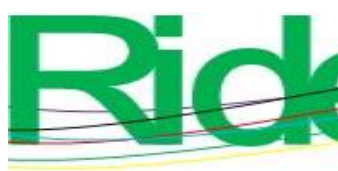
Revista Iberoamericana para la
Investigación y el Desarrollo Educativo
ISSN $2007-7467$

\section{Referencias}

Baptista, P., Almazán, A., Loeza, C., López, V. y Cárdenas, J. (2020). Encuesta nacional a docentes ante el covid-19. Retos para la educación a distancia. Revista Latinoamericana de Estudios Educativos, 50, 41-88. Recuperado de https://doi.org/10.48102/rlee.2020.50.ESPECIAL.96

Burgos, C., Vázquez, E., López, E. y Adaos, R. (2021). Proyecto DIFPRORET: análisis de las dificultades, propuestas y retos educativos ante el covid-19. International Journal of Educational Research and Innovation (IJERI), 15, 17-34. Doi: https://doi.org/10.46661/ijeri.5145

Cabero, J. y Valencia, R. (2021). Y el covid-19 transformó al sistema educativo: reflexiones y experiencias por aprender. International Journal of Educational Research and Innovation (IJERI), 15, 217-227. Doi: https://doi.org/10.46661/ijeri.5246

Cáceres, K. (2020). Educación virtual: creando espacios afectivos, de convivencia y aprendizaje en tiempos de covid-19. CienciAmérica, 9(2), 1-7. Recuperado de http://cienciamerica.uti.edu.ec/openjournal/index.php/uti/article/view/284/424

Cacheiro, M. (2014). Educación y tecnología: estrategias didácticas para la integración de las TIC. Madrid: Editorial UNED.

Carmona, D. y Morales, H. (2021). Retos de la pedagogía en los tiempos de covid-19. Archivos en Medicina Familiar, 23(2), 59-64. Recuperado de https://www.medigraphic.com/pdfs/medfam/amf-2021/amf212a.pdf

Cervantes, E. y Gutiérrez, P. (2020). Resistir la covid-19. Intersecciones en la educación de la Ciudad Juárez, México. Revista Internacional de Educación para la Justicia Social, 9(3), 7-23. Doi: https://doi.org/10.15366/riejs2020.9.3.001

Chaves, A. (2017). La educación a distancia como respuesta a las necesidades educativas del siglo XXI. Revista Academia y Virtualidad, 10(1), 23-41. Doi: https://doi.org/10.18359/ravi.2241

Cifuentes, J. (2020). Docencia online y covid-19: la necesidad de reinventarse. Revista de Estilos de Aprendizaje, 13, 115-127. Recuperado de http://revistaestilosdeaprendizaje.com/article/view/2149/3248

Consejo Nacional de Evaluación de la Política de Desarrollo Social [Coneval]. (2018). Medición de la pobreza. Coneval: México. Recuperado de https://www.coneval.org.mx/Medicion/MP/Paginas/Pobreza-2018.aspx. 


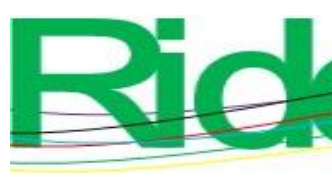

Revista Iberoamericana para la Investigación y el Desarrollo Educativo ISSN $2007-7467$

Denzin, N. y Lincoln, Y. (2012). El campo de investigación cualitativa. Manual de investigación cualitativa (vol. 1). España: Gedisa editorial.

Díaz-Barriga, F. y Hernández, G. (2010). Estrategias docentes para un aprendizaje significativo. Una interpretación constructiva. México: McGraw Hill.

Ducoing, P. (2020). Una expresión de la desigualdad en educación básica durante la emergencia sanitaria: el caso de una alumna. En Instituto de Investigaciones sobre la Universidad y la Educación de la UNAM (coord.), Educación y pandemia. Una visión académica (pp. 55-64). México: Universidad Nacional Autónoma de México. Recuperado de http://www.iisue.unam.mx/nosotros/covid/educacion-y-pandemia

Ferreiro, R. (2012). Cómo ser mejor maestro. El método ELI. México: Editorial Trillas.

García, J. (2012). La educación emocional, su importancia en el proceso de aprendizaje. Revista Educación, 36(1), 1-24. Recuperado de https://www.redalyc.org/pdf/440/44023984007.pdf

Gazca, J. (2020). Implicaciones del coronavirus covid-19 en los procesos de enseñanza en la educación superior. Revista Iberoamericana para la Investigación y el Desarrollo Educativo, 11(21), 1-30. Doi: https://doi.org/10.23913/ride.v11i21.753

Heedy, C. y Uribe, M. (2008). La educación a distancia: sus características y necesidad en la educación actual. Educación, 17(33), 7-27. Recuperado de http://revistas.pucp.edu.pe/index.php/educacion/article/view/1532

Hernández, R., Fernández, C. y Baptista, P. (2010). Metodología de la investigación. (5. ed.). Perú: McGraw-Hill:

Instituto Nacional de Estadística y Geografía [Inegi]. (2020). Estadísticas a propósito del día mundial del internet (17 de mayo) datos nacionales. Recuperado de https://www.inegi.org.mx/contenidos/saladeprensa/aproposito/2020/EAP_Internet2. pdf

Izcara, S. (2014). Manual de investigación cualitativa. México: Editorial Fontamara.

Maxwell, I. (2004). Using qualitative methods for causal explanations. California: Thousand Oaks.

Mendoza, D. (2018). Influencias cognoscitivas de la tecnología de información y comunicación en el aprendizaje de la matemática. Innova, 3(9), 1-17. Recuperado de http://revistas.uide.edu.ec/index.php/innova/article/view/446 


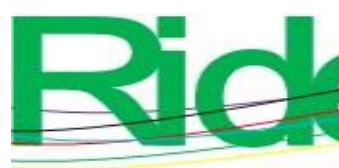

Revista Iberoamericana para la
Investigación y el Desarrollo Educativo
ISSN $2007-7467$

Moreira, P. (2019). El aprendizaje significativo y su rol en el desarrollo social y cognitivo de los adolescentes. Rehuso, 4(2), 1-12. Recuperado de https://revistas.utm.edu.ec/index.php/Rehuso/article/view/1845

Moreno, S. (2020). La innovación educativa en los tiempos del coronavirus. Salutem Scientia $\begin{array}{llll}\text { Spiritus, } & 6(1), & \text { 14-26. } & \text { Recuperado }\end{array}$ https://revistas.javerianacali.edu.co/index.php/salutemscientiaspiritus/article/view/2 290

Muñoz, J. y Lluch, L. (2020). Educación y covid-19: colaboración de las familias y tareas escolares. Revista Internacional de Educación para la Justicia Social, 9(3), 1-17. Recuperado de https://revistas.uam.es/riejs/article/view/12182

Parra, S., Gómez, M. y Pintor, M. (2015). Factores que inciden en la implementación de las TIC en los procesos de enseñanza-aprendizaje en 5. de Primaria en Colombia. Revista Complutense de Educación, 26, 197-213. Recuperado de https://revistas.ucm.es/index.php/RCED/article/view/46483

Sánchez, M., Martínez, A., Torres, R., De Agüero, M., Hernández, A., Benavides, L., James, C. y Rendón, V. (2020). Retos educativos durante la pandemia covid-19: una encuesta a profesores de la UNAM. Revista Digital Universitaria, 21(3), 1-24. Doi: http://doi.org/10.22201/codeic.16076079e.2020.v21n3.a12

Secretaría de Educación Pública [SEP]. (2020a). Aprende en casa. Recuperado de https://aprendeencasa.sep.gob.mx/

Secretaría de Educación Pública [SEP]. (2020b). Estrategia de educación a distancia: transformación e innovación para México “Propuesta integral frente al COVID-19”. Recuperado de https://septlaxcala.gob.mx/comunicado/estrategia_para_educacion_a_distancia_cont igencia_covid_19.pdf

Tobón, M. (2010). Formación integral y competencia, pensamiento complejo, diseño curricular y didáctica. Colombia: ECOE.

Umaña, A. (2020). Educación superior en tiempos de covid-19: oportunidades y retos de la educación a distancia. Innovaciones Educativas, 22, 36-49. Doi: https://doi.org/10.22458/ie.v22iEspecial.3199

Vasilachis, I. (2006). Estrategias de investigación cualitativa. España: Gedisa.

Vela, P., Ahumada, V. y Guerrero, J. (2015). Conceptos estructurantes de la educación a distancia. Revista de Investigaciones UNAD, 14(1), 115-149. 


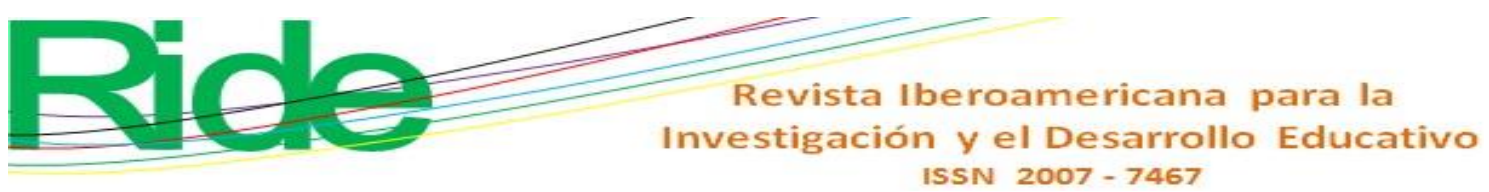

Zavala, M., González, I. y Vázquez, M. (2020). Modelo de innovación educativa según las experiencias de docentes y estudiantes universitarios. Revista Iberoamericana para la Investigación y el Desarrollo Educativo, 10(20), 1-25. Doi: https://doi.org/10.23913/ride.v10i20.590 